\title{
Der Non-Suizid-Vertrag auf dem Prüfstand
}

\author{
Lisa Gerstl
}

Online publiziert: 3. Dezember 2019

(C) Der/die Autor(en) 2019

Zusammenfassung Die Behandlung suizidaler Patien_innen birgt eine Komponente, die nicht in jeder Psychotherapie zum Ausdruck kommt: die Bedrohung des Lebens der Patient_innen sowie damit einhergehend die Angst der Behandler_innen um die Patient_innen bzw. vor rechtlichen Folgen. Auf Grund dessen kommen in solchen Behandlungen oft Non-Suizid-Verträge (NSV) zum Einsatz. Die Intention solch einer Intervention kann in der Aufrechterhaltung des therapeutischen Prozesses und in der Absicherung von Leib und Leben der Patient_innen gesehen werden. Im Rahmen des vorliegenden Artikels wird der Ausgangspunkt sowie die Verbreitung der Intervention NSV aufgezeigt. Anschließend werden ausgewählte Studien präsentiert, die Wirkfaktoren (Haupt-Wirkung, Neben-Wirkung, Wechsel-Wirkung und Schäden) ausgearbeitet und Implikationen für die Praxis, unter Anlehnung an alternative Interventionen bei der Arbeit mit suizidalen Patient_innen, gegeben. Die Diskussion der Ergebnisse ausgewählter Studien zeigt, dass der Einsatz eines NSV kritisch gesehen werden muss, da er oft unreflektiert erfolgt und NSV sich förderlich, aber genauso schädlich auf die Suizidalität auswirken können.

\section{Schlüsselwörter Suizidalität · Non-Suizid-Vertrag ·} Suizidverhütung $\cdot$ Krisenintervention

\section{Rethinking the use of non-suicide-contracts}

Summary The treatment of suicidal patients bears a component that is not subject to every psychotherapy: the threat of the patient's life. Accompanied by fear of the practitioner for the patient or legal con-

\section{Gerstl ( $\bowtie)$}

Währinger Gürtel 154/12, 1090 Wien, Österreich

lisa.gerstl.it@gmail.com sequences. Due to this, non-suicide-contracts (NSC) are often used. The intention of such an intervention can be seen in the maintenance of the therapeutic process and in the protection of life and limb of the patient. The initial usage of NSC as well as it's prevalence are shown. Furthermore, selected studies are presented, factors are mapped out (main effect, side effect, change effect and damage) and implications for the practice of therapists are given. Those are based on alternative interventions to NSC. The discussion of selected studies shows that the use of a NSC must be viewed critically, as it's use is often unconsidered. Moreover NSC can be beneficial as well as harmful to suicidal patients.

Keywords Integrative Therapy $\cdot$ Suicidality $\cdot$ NonSuicide-Contract · Suicide prevention · Crisis intervention

Menschen, die beim Aufsuchen von ambulanten und stationären psychotherapeutischen oder psychiatrischen Angeboten Suizidalität geäußert haben, sind bis dato häufig mit Vereinbarungen in Kontakt gekommen, die die Distanzierung zum Suizid zum Thema hatten - sogenannte Non-Suizid-Verträge (NSV).

Im vorliegenden Artikel werden ausgehend von der Thematik Psychotherapie mit suizidalen $\mathrm{Pa}$ tient_innen, der Ursprung und die Entwicklung von NSV aufgezeigt und auf deren Nützlichkeit überprüft. Wirkfaktoren werden ausgearbeitet und abschließend werden Implikationen für die Praxis von freiberuflichen Behandler_innen gegeben.

\section{Psychotherapie mit suizidalen Patient_innen}

In der Suizidprävention wie in der Krisenintervention geht es bei vorherschender Suizidalität immer um 
rasche Intervention (Wolfersdorf und Etzersdorfer 2011). Es ist wichtig, individuelle Herangehensweisen $\mathrm{zu}$ entwickeln, die der aktuellen Situation und der hilfesuchenden Person entsprechen. Es gibt keine allgemeine Herangehensweise, die Handlungserfolg verspricht. Gängige Praxis und wesentlich ist, nicht die einzige Ansprechperson für diese Patient_innen zu sein, sondern im Team mit Psychiater_innen, Kriseninterventionsstellen bzw. anderen Betreuungseinrichtungen $\mathrm{zu}$ arbeiten (Dorrmann 2009; Sonneck et al. 2016).

Wenden sich suizidale Patient_innen an Psychotherapeut_innen, so kann man eine gewisse Ambivalenz bezüglich der Selbsttötungsabsichten und -gedanken annehmen (Aldenhoff 2017). In den Psychotherapeut_innen wird dann eine Hilfsperson gesehen, die Hilfsangebote erbringen soll. Reichel (2018) spricht sich dafür aus, als Psychotherapeut_in einen Umgang mit suizidalen Patient_innen zu pflegen, der darauf baut, das Gegenüber zu verstehen und zu bestätigen und gleichzeitig als Repräsentant_in für den Lebenswillen zu stehen.

Die Herausforderung für Behandler_innen in der Arbeit mit suizidalen Patient_innen liegt darin, den therapeutisch wirksamsten Weg zu gehen, der gleichzeitig so wenig restriktiv wie möglich sein soll. Ist eine suizidale Einengung bereits gegeben, sind der Aufbau, die Pflege und ein Erreichen von Stabilität und Sicherheit in der psychotherapeutischen Beziehung von großer Bedeutung (Bronisch 2014, 2017). Sonneck et al. (2016) halten fest, dass nur die Beziehung zwischen Psychotherapeut_innen und Patient_innen eine Einengung zu lockern vermag. Sie kann als stellvertretende Hoffnung für die Patient_innen gesehen werden, die für diese von großer Bedeutung ist. Von Wichtigkeit ist aber auch die Festigung bzw. der Rückhalt des sozialen Systems, damit auch dieses $\mathrm{zu}$ einem Hoffnungsträger werden kann und das Bezugs-/ Entlastungssystem nicht nur innerhalb professioneller Versorgung liegt.

Auf Grund der Garantenstellung der Behandler_innen und der damit verbundenen Verantwortung werden bei der Arbeit mit suizidalen Patient_innen bei Behandler_innen oft Ängste ausgelöst. Vom Einsatz von NSV zur Minimierung der Ängste der Behandler_innen ist Abstand zu nehmen. Stattdessen ist auf Transparenz der ausgelösten Gefühle zu setzen, was auch als beziehungsfördernd angesehen werden kann (Sonneck et al. 2016).

Reichel (2018) hält für die Situation nach einem Suizidversuch oder einer Selbstverletzung fest, dass Fassungslosigkeit und Sprachlosigkeit oft die einzig „ehrliche Reaktion“ (S. 218) darstellen. Es gilt in dem Fall keine Warum-Fragen zu stellen und keine Vorwürfe zu machen, da sie einen neuen Suizid(versuch) nähren können. Es wird konstatiert, dass es wichtig sei, Freude darüber zu vermitteln, dass der_die Andere noch lebt. Wichtig ist die ehrliche und authentische Anteilnahme.
Sonneck (2001) weist darauf hin, den Fokus nicht darauf zu legen, wie man einen Menschen davon abhalten kann, sich zu suizidieren, sondern das Augenmerk darauf zu legen, wie sich die Lebensumstände ändern müssen, um das Leben (wieder) als lebenswert zu empfinden. Damit wird der Blick in die Zukunft gelegt, Veränderungen, die getroffen werden müssen, können sichtbar werden und Ressourcen erschlossen werden.

\section{Der Non-Suizid-Vertrag}

Im Fokus der Aufmerksamkeit steht anschließend der Ursprung der Intervention NSV sowie die Entwicklung hin zu einer gänigen Intervention.

Der ursprüngliche NSV, wie er von Drye et al. (1973) verfasst wurde, lautet: „No matter what happens, I will not kill myself, accidentally or on purpose, at any time“ (S. 172).

Durch den Einsatz eines NSV sollen der Argumentation Drye et al. (1973) folgend, Behandler_innen mit ihnen bereits bekannten Patient_innen ein gemeinsames Ziel, nämlich die Sicherung des Lebens, haben. Darüber hinaus soll das Sprechen über Suizidalität ermöglicht werden und sich positiv auf die therapeutische Beziehung auswirken. Die Wirksamkeit ist dadurch erläutert worden, dass es bei 600, mit Patient_innen abgeschlossenen, NSV in der Zeitspanne von 5 Jahren zu keinem Suizid kam.

Die Forscher_innen haben jedoch nicht die relativen Zahlen bereitgestellt, wie viele bzw. mit wem NSV vereinbart worden sind. Unter anderem ist keine statistische Analyse durchgeführt und sind keine Kriterien für den Grad der Suizidalität festgelegt worden, weshalb die Reproduzierbarkeit nicht möglich ist (Drew 2001; Lee und Bartlett 2005; Page und King 2008; Range et al. 2002).

Drew (2001) streicht heraus, dass Drye, Goulding und Goulding den NSV auf einem transaktionsanalytischen Ansatz aufgebaut haben. Die Autor_innen haben den NSV als Stellungnahme des erwachsenen Ego-States gesehen, der dafür verantwortlich ist, Verantwortung zu übernehmen und für Sicherheit für Leib und Leben zu sorgen.

In den 1970er Jahren haben sich problemzentrierte bzw. lösungsorientierte Therapieansätze entwickelt, was als Erklärungsansatz für die Etablierung von NSV gesehen werden kann. Behandler_innen haben eine Übereinstimmung zwischen dem Einsatz von NSV und kognitiver bzw. dialektisch-behavioraler Therapie bemerkt, weil in beiden die Zustimmung der Patient_innen gegeben wurde, in ihren Themenbereichen trainiert $\mathrm{zu}$ werden, i.e. sich neue Verhaltensmuster anzueignen und diese auch eigenverantwortlich außerhalb der Therapie zu üben. Darüber hinaus sind $\mathrm{zu}$ der Zeit Krisentelefone bzw. Krisenzentren aufgekommen, in denen NSV von nun an auch bei Erstkontakten eingesetzt worden sind (Edwards und Harries 2007). 
Aus psychodynamischer Sicht führen NSV oft dazu, dass die mit der Suizidalität verknüpften Themen sich in der Beziehung zu den Psychotherapeut_innen wiederholen bzw. aktualisiert werden. Kind (2011, S. 188) hält diesbezüglich zwei gängige Formen fest:

- Der Therapeut tritt in gewisser Weise durch den Pakt dem Patienten zu nahe, bedroht seine Abgrenzungsbemühungen und seine Autonomie.

- Er versucht jenen Bereich, mit dem der Patient sich gefährdet, unter Kontrolle zu bringen, und aktualisiert dadurch die Auslieferungs- und Ohnmachtsgefühle des Patienten.

Es ist damit zu rechnen, dass ein Pakt gebrochen wird, vor allem in der Therapie mit chronisch suizidalen Patient_innen. Ein NSV trifft Patient_innen „in Bereichen schwach ausgebildeter psychischer Funktionen“ (Kind 2011, S. 188). Das Brechen der Abmachungen soll nicht als Problem für den Verlauf der Therapie gesehen werden, sondern fordert einen entsprechenden Umgang mit dem Verhalten (Kind 2011). Ein daraus resultierendes Ziel kann das Mitteilen von sich aufdrängenden Suizidgedanken, -ideen oder -impulsen sein oder wenn diese nicht mehr kontrolliert werden können (Kind 2011). Stanley et al. (2017) betonen den suizidalen Drang in den Fokus zu nehmen, um das persönliche Verständnis von Suizidalität zu schärfen.

Lee und Bartlett (2005) haben in den Death Studies auf Basis wissenschaftlicher Artikel eine theoretische Abhandlung von positiven und negative Aspekten von NSV ausgearbeitet. Ein NSV kann den Autor_innen nach als entlastend wahrgenommen werden, wenn das Gefühl, dass das Leben außer Kontrolle ist, auftritt. Der NSV kann, besonders bei Menschen mit Persönlichkeitsstörungen, das Gefühl vermitteln, dass Kontrolle möglich ist. Eine mögliche Auswirkung eines NSV kann sein, dass Patient_innen ermutigt werden, über Suizidalität zu sprechen. Ebenso kann die therapeutische Beziehung negativ getrübt werden, wenn Patient_innen auf Grund eines NSV nicht mehr über ihre Suizidalität sprechen, aus Sorge, damit den NSV zu verletzen. Die Folge können Kommunikationsbarrieren sein, in weiterer Folge das Leugnen von Suizidalität, was eine Gefahr für die Patient_innen darstellt. Manche Patient_innen können aber auch den Eindruck bekommen, dass der NSV nur eine Entlastungsfunktion für Behandler_innen hat. Der NSV darf nicht als Begutachtungsinstrument gesehen werden. Die Beurteilung der Suizidalität hat über längere Zeit zu erfolgen und muss diagnostischen Kriterien folgen. Ein NSV ist eine Momentaufnahme, während die Begutachtung als Prozess zu verstehen ist.

NSV werden von einigen Expert_innen verwendet, obwohl sie nur spärlich erforscht sind. Die Annahme drängt sich auf, dass die Verwendung auf Eindrücken oder Erfahrungswerten basiert (Range et al. 2002). Der nächste Abschnitt befasst sich mit der Darstellung von Studienergebnissen.

\section{Die Effektivität von Non-Suizid-Verträgen}

Studien zur Nützlichkeit von NSV im klinischen Alltag bzw. in der Praxis zeichnen mehrheitlich das Bild der fehlenden Beweisbarkeit von Suizidprophylaxe durch den Einsatz eines NSV (Kelly und Knudson 2000; Reid 1998; Stanford et al. 1994). Der Autorin ist keine randomisierte Kontrollstudie zu der aus 1973 von Drye et al. bekannt (Stanley und Brown 2012). Folgend werden zwei Studien aus 2008 und 2017 vorgestellt.

Page und King (2008) haben in Kanada im Rahmen einer Studie evaluiert, in welchem Ausmaß NSV bei niedergelassenen Therapeut_innen zum Einsatz kommen. 516 Therapeut_innen mit unterschiedlichen Grundberufen sind zu ihrem Gebrauch bzw. ihren Erfahrungen mit NSV befragt worden. Die Umfrage ist von 312 Therapeut_innen retourniert worden und hat folgende Ergebnisse gebracht: $83 \%$ der Befragten haben einen NSV verwendet (die Hauptzahl davon Therapeut_innen ohne medizinischen Grundberuf), obwohl nur $40 \%$ eine Einschulung in die Verwendung eines NSV erhalten haben.

Orientiert hat sich der Gebrauch von NSV an der Einschätzung der Suizidalität. $31 \%$ der Therapeut_innen, die einen NSV verwendet haben, haben angegeben, dass mindestens ein_e Patient_in, trotz des Einsatzes eines NSV, einen Suizidversuch unternommen bzw. Suizid begangen hat. Bei Nichtzustandekommen eines NSV hat der Großteil der Befragten den Versuch unternommen, die Patient_innen stationär unterzubringen (Page und King 2008).

Als Grund für die Verwendung des NSV haben die Therapeut_innen angegeben, dass er Sorge und Fürsorge zeige. Einerseits hat die Hälfte der Therapeut_innen geglaubt, dass ein NSV die Suizidwahrscheinlichkeit reduziere, während gleichzeitig mehr als die andere Hälfte der Befragten geglaubt hat, dass er die Angst der Therapeut_innen reduziere. Gespalten haben sich die Befragten in der Frage des Haftungsschutzes durch einen Non-Suizid-Vertrag bei Suizid gezeigt. Die Studienautor_innen folgern, dass die Befragten glauben der Einsatz von NSV sei sinnvoll und plädieren für intensiveres Training im Umgang mit NSV und rechtlichen Aspekten (Page und King 2008).

2017 wurde eine randomisierte klinische Vergleichsstudie von Bryan et al. (2017) durchgeführt. Ziel der Studie war ein Vergleich der Effektivität von Krisenreaktionsplänen (= KRP) und NSV bei Suizidgedanken oder suizidalem Verhalten. Die Studie wurde mit 97 aktiven Soldat_innen der amerikanischen Armee durchgeführt, die einen Notfalltermin auf Grund von krisenhaftem Verhalten gehabt haben. Mit 32 Personen wurde ein NSV, mit 32 Personen ein KRP und mit 33 Personen ein erweiterter KRP abgeschlossen.

Ein KRP, wie er im Laufe der Studie durchgeführt wurde, zeichnet sich dadurch aus, dass er auf Scheckkartenformat notiert, stets von den Patient_innen mit- 
geführt wird und folgende Elemente enthält (Bryan et al. 2017) [Übersetzung der Autorin]:

- persönliche Warnsignale, um sie zu erkennen

- persönlich hilfreiche Bewältigungsmechanismen bzw. Skills

- eingeweihte Bezugsperson(en) kontaktieren bzw. Kontaktaufnahme mit dem sozialen Netz, um Unterstützung zu bekommen

Ein KRP ist als eine Möglichkeit zu verstehen, Patient_innen eine Anleitung zu geben, was sie während einer suizidalen Krise tun sollen. Abgedeckt werden das Erkennen von Risikobereichen und darauf bezogene hilfreiche Handlungen, was im Weiteren zur Souveränitätssteigerung der Patient_innen beitragen kann, wenn sie ihre Selbstwirksamkeit erleben (Bryan et al. 2017).

Die Ergebnisse haben gezeigt, dass es keinen signifikanten Unterschied zwischen einem erweiterten KRP und einem KRP gibt, weshalb die Ergebnisse $\mathrm{zu}$ einer Kategorie mit 65 Personen zusammengefügt worden sind. Im Nachuntersuchungszeitraum von 6 Monaten haben drei Teilnehmer_innen (4,9\%), die einen KRP bekommen haben und fünf Teilnehmer_innen (19\%), die einen NSV erhalten haben, einen Suizidversuch unternommen.

Patient_innen mit einem KRP weisen demnach innerhalb des Nachuntersuchungszeitraums eine um $76 \%$ geringere Wahrscheinlichkeit auf, einen Suizidversuch zu vollziehen. Neben der geringeren Anzahl von Suizidversuchen bei denjenigen Teilnehmer_innen mit KRP konnten ebenso rascher abnehmende Suizidgedanken und eine geringere Anzahl an stationären Aufenthaltstagen verzeichnet werden, was die Autor_innen hat folgern lassen, dass der Krisenreaktionsplan effektiver ist als der NSV (Bryan et al. 2017).

Bryan et al. (2017) erwähnen, dass der Einsatz von KRP (sowie NSV) auf Grundlage von Glaubenssätzen bzw. Erfahrungswerten der Behandler_innen beruht. Für die Interventionsform KRP liegen noch keine empirischen Daten vor, weshalb zukünftig zu untersuchen sein wird, welche Komponenten eines KRP das Suizidrisiko senken.

Die Studienergebnisse unterstreichen einerseits das Phänomen der Beliebtheit von NSV bei den Behandler_innen, während andererseits andere Interventionsformen bessere Ergebnisse in der Suizidprophylaxe aufweisen. Es ist anzunehmen, dass weitere Parameter, wie etwa Tragfähigkeit der therapeutischen Beziehung oder Halt im sozialen Netz, die Effiktivität beeinflussen.

\section{Die Wirkfaktoren von Non-Suizid-Verträgen}

In Anlehnung an Leitners Einteilung der Wirkfaktoren von Psychotherapie (2011) werden anschließend die Kategorien Haupt-, Neben-, Wechselwirkungen und
Schäden herangezogen, um sie in Bezug zu NSV zu stellen.

Als Haupt-Wirkungen von NSV sind folgende Punkte identifizierbar:

- Der Abschluss eines NSV bei aufrechter, stabiler Beziehung kann und soll die Selbstkontrolle der Patient_innen stärken (Lazic et al. 2015).

- Der Abschluss eines NSV bei ambivalenter Einstellung der Patient_innen zu ihrer Suizidalität kann die Selbstregulation stärken (Lazic et al. 2015).

- Bei plötzlicher Suizidalität und der Notwendigkeit, sofort zu reagieren, haben sich NSV als die hilfreichste und schnell verfügbare Intervention etabliert (Edwards und Harries 2007).

Als Neben-Wirkungen von NSV sind folgende Punkte identifizierbar:

- Der Abschluss eines NSV kann die therapeutische Beziehung stärken, wenn Patient_innen das Gefühl haben, die Behandler_innen sind wirklich an ihrem Wohl interessiert (Lee und Bartlett 2005).

- Der Abschluss eines NSV kann die therapeutische Beziehung gefährden, wenn Auslieferungs- und Ohnmachtsgefühle der Patient_innen ausgelöst werden (Kind 2011).

Als Wechsel-Wirkungen von NSV sind folgende Punkte identifizierbar:

- Oft wird vom Abschluss eines NSV ein Sicherheitsaspekt für die Patient_innen erwartet, der jedoch nicht angenommen werden kann. Das Versprechen kann durch die „Schwere der Erkrankung..., durch im Moment des Versprechens unvorhersehbare, unerwartete Ereignisse und ... [auf Grund der] individuell variable[n] Handhabung der Verbindlichkeit“ beeinflusst werden (Lazic et al. 2015, S. 662).

- Der NSV wird überbewertet und auf Grund dessen flächendeckend unreflektiert zum Einsatz gebracht (Jacobs et al. 2010).

- Wechselwirkungen entstehen, wenn Behandler_innen den NSV als persönlichen Schutz vor den Rechtsfolgen einsetzen, obwohl sie denken, dass ein NSV wenig hilfreich zur Verhinderung von Suizidalität ist (Edwards und Sachmann 2010).

Ein NSV hat keinen gültigen Rechtscharakter und bildet gängiger Meinung nach keinen entlastenden Schutz im Sinne eines Beweisdokuments bzw. rechtsgültigen Vertrags bei einer Anklage. (Schiller (2011) ist als einer der wenigen zu nennen, der den Abschluss eines NSV als positiv für das Gerichtsverfahren im Rahmen der (!) Ärzt_innenhaftung einstuft.) Schutz bei einer Anklage kann nur ausführliche Dokumentation sowie ein gründliches und prozessuales Befunden der Suizidalität bieten (Edwards und Sachmann 2010; Jacobs et al. 2010; Lee und Bartlett 2005; Page und King 2008).

- Es kann sein, dass bei Abschluss eines NSV die Wachsamkeit bzw. das Wahrnehmen der Behand- 
ler_innen in puncto Anzeichen oder Andeutungen von Suizidalität nachlässt (Jacobs et al. 2010).

- Ein NSV ist nur so verlässlich, wie die bestehende therapeutische Beziehung (Jacobs et al. 2010; Lazic et al. 2015; Page und King 2008).

Als Schäden von NSV sind folgende Punkte identifizierbar:

- Die Funktion von NSV hat sich davon, Teil einer Befundung und Diagnostik zu sein, zu einer Intervention zum Schutz gegen Behandlungsfehler verändert (Edwards und Harries 2007).

- Ein Schaden kann entstehen, wenn Behandler_innen den NSV verwenden, um einen Umgang mit den eigenen Ängsten zu finden bzw. diese zu verringern. Diese Ängste können sich um das Wohl der Patient_innen drehen, aber auch um Konsequenzen in Bezug auf die Ausübung der Profession der Behandler_innen. Oft spüren Patient_innen die Angst bzw. Sorge der Behandler_innen und geben das Versprechen, am Leben zu bleiben, im Rahmen eines NSV nur, um die Behandler_innen zu beruhigen (Lazic et al. 2015; Lee und Bartlett 2005).

- Ein Schaden kann verursacht werden, wenn ein NSV abgeschlossen wird, um dadurch Beziehung herzustellen bzw. zu stärken (Lazic et al. 2015).

- Ein Schaden kann entstehen, wenn keine ausführliche, prozessuale Befundung der Suizidalität gemacht wird, weil ein NSV abgeschlossen wird. Ebenso ist es schädlich, keinen Behandlungsplan festzulegen (Edwards und Harries 2007; Jacobs et al. 2010).

- Ein Schaden kann hervorgerufen werden, wenn Patient_innen sich auf Grund eines NSV nicht mehr trauen, Äußerungen über Suizidgedanken oder -impulse zu tätigen (Lee und Bartlett 2005; Stanley und Brown 2012).

- Ein Schaden kann hervorgerufen werden, wenn sich auf Grund der Intervention durch NSV bei Patient_innen ein Gefühl von Autorität seitens der Behandler_innen einstellt (Kind 2011).

- Ein Schaden kann entstehen, wenn Patient_innen wissen, dass sie, wenn sie den NSV nicht unterschreiben, von den Behandler_innen gebeten werden, eine stationäre Behandlung zu beginnen bzw. von ihnen untergebracht werden (Page und King 2008).

- Die Clinical Practice Guidelines for the management of adult deliberate self-harm of the Royal Australian and New Zealand College of Psychiatrists identifizieren NSV als potentiell schädlich (Edwards und Harries 2007).

Die Abhandlung macht sichtbar, dass ein Ungleichgewicht zwischen Haupt-Wirkfaktoren und Schäden vorliegen. Die Wirkweise von stark von der Beziehung zu den Patient_innen, gleichzeitig von deren Persönlichkeitsstruktur und darüber hinaus vom persönlichen Umgang der Behandler_innen mit der Angst um ihre Patient_innen abhängig. Dadurch wird der NSV als „Standardintervention“ in Frage gestellt. Im Folgenden werden die gewonnenen Erkenntnisse $\mathrm{zu}$ Implikationen für die Praxis ausgearbeitet.

\section{Implikationen für die Praxis}

Die Frage nach der sicheren Herstellung von Schutz für suizidale Patient_innen bleibt mit der Intervention NSV unbeantwortet. Franklin et al. (2017) führten eine Metaanalyse von 365 Studien der vergangenen 50 Jahre durch, die das Ziel hatten, die suizidale Neigung (Gedanken und Handlungen) abzuschätzen bzw. auf lange Sicht vorherzusagen. Laut den Studienautor_innen waren die Forschungsdesigns zu homogen, das Zusammenspiel multipler Risikofaktoren wurde nicht berücksichtigt. Dabei stießen sie auf die unerwartete Erkenntnis, dass die Vorhersagbarkeit von Risikofaktoren nur wenig größer sei, als der Zufall der richtigen Einschätzung.

Klonsky (2019) betont, es sei eine Fehlinterpretation zu glauben, dass eine gültige Theorie eine Vorhersage von zukünftigen Ereignissen erlaube. Fehleinschätzungen bzw. -prognosen würden, der Argumentation folgend, die Gültigkeit und Nützlichkeit der Theorie widerlegen.

Gerade weil bis dato kein Modell entwickelt werden konnte, das Suizidalität erwiesener Maßen hemmen oder verringern kann, wird abschließend ein Entwurf von Implikationen angeführt, der den Fokus einerseits auf die persönlichen Gründe für das am Leben bleiben rückt und andererseits die Handlungsfähigkeit der $\mathrm{Pa}-$ tient_innen sichtbar machen soll.

Die angeführten Implikationen zur Suizidverhütung sind mit anderen bekannten Interventionen vergleichbar. So werden Ähnlichkeiten mit Notfallplänen (Dorrmann 2009), crisis response planning (Bryan et al. 2017), SPI (Savety Plan Intervention) (Stanley und Brown 2012), AIM-SP (Assess, Intervene and Monitor for Suicide Prevention model) (Brodsky, Spruch-Feiner und Stanley 2018) und management plan (Lee und Bartlett 2005) sichtbar. Es sind dies Interventionen, in denen der Fokus auf der Steigerung der Souveränität liegt, indem Faktoren zur Selbsthilfe und Pläne zur Durchführung in Krisensituationen erarbeitet werden.

Der Entwurf hat keinen Anspruch auf Vollständigkeit und soll als Anregung dienen. Die Ordnung der zu besprechenden Themenkomplexe folgt keiner Hierarchie und kann frei verändert werden. Auch die Aufzählungen innerhalb der einzelnen Themenkomplexe sind nicht als Empfehlung in der gegebenen Reihenfolge zu lesen. Die Anregungen sind als Möglichkeit zu sehen; mit den Patient_innen soll ausgearbeitet werden, welche Punkte in welcher Reihenfolge für sie am dienlichsten sind. 


\section{Rahmenbedingungen}

Die Voraussetzungen für die Vereinbarung von Rahmenbedingungen sind ident $\mathrm{zu}$ jenen beim Abschluss eines NSV.

- Es muss das freiwillige Einverständnis von Seiten der Patient_innen geben sein.

- Ebenso muss die Urteils- und Einsichtsfähigkeit gegeben sein, was bedeutet, dass Patient_innen sich auf eine Vereinbarung dieser Art einlassen können (Paktfähigkeit).

- Nicht möglich ist das Vereinbaren von Rahmenbedingungen demnach für Menschen mit Realitätsverlust bei depressiven oder schizophrenen Psychosen, bei Einfluss von Suchtmitteln, bei eingeschränkter Impulskontrolle mit autoagressivem und selbstschädigendem Verhalten (Bronisch 2014).

Absprachen bzw. Vereinbarungen mit den Behandler_innen sollen hinsichtlich der Kontaktaufnahme in Krisen getroffen werden. Dabei sollen folgende Aspekte geklärt werden:

- Zu welcher Uhrzeit ist der_die Behandler_in telefonisch/per E-Mail erreichbar.

- Die Dauer eines Krisengesprächs am Telefon/EMail-Verkehr.

- In welchem Zeitabstand zum Telefonat/E-MailKontakt bekommen Patient_innen einen Krisentermin.

- Wann werden Patient_innen an eine Institution wie einem Kriseninterventionszentrum bzw. einer psychiatrischen Ambulanz überwiesen. Dabei ist es wichtig vorab zu vereinbaren, ob Patient_innen von den Behandler_innen oder wichtigen Bezugspersonen begleitet werden.

- Wann werden Patient_innen auf Grund von Selbstgefährdung in eine Psychiatrie eingewiesen. Abzuklären ist, ob die Patient_innen selbst die Rettung rufen oder die Behandler_innen.

- Festlegen eines Kontakts, an den sich Patient_innen wenden, wenn der_die Behandler_in nicht erreichbar ist.

Diese Aspekte sind für Patient_innen in Krisensituationen notwendig $\mathrm{zu}$ erinnern, weshalb empfohlen wird, sie im Rahmen einer Therapieeinheit auf ein Kärtchen zu notieren. Das Kärtchen soll an einem bestimmten Platz angebracht werden bzw. von den Patient_innen in der Geldbörse/dem Rucksack/etc. mitgeführt werden. Ein Formulierungsvorschlag:

Ich spüre einen inneren Druck größer werden/ merke Suizidimpulse, -gedanken, etc., ich erinnere mich, dass ich nicht alleine damit zurechtkommen muss, sondern

- meine_n Therapeut_in + Name+Telefonnummer

- meine_n Freund_in + Name + Telefonnummer

- meine Mutter/meinen Vater/Geschwister + Name+ Telefonnummer
- meine_n Nachbar_in + Name + Telefonnummer

- meine_nLehrer_in/Vereinsleiter_in/Bandkolleg_innen/usw. + Name + Telefonnummer

- eine Institution wie z.B. das Kriseninterventionszentrum + Telefonnummer

anrufen/besuchen/anschreiben kann und mich darauf verlassen kann, um einen Spaziergang/ein Gespräch/ein Verbringen gemeinsamer Zeit im Schweigen/etc. bitten zu können.

\section{Therapeutische Schwerpunkte}

Im Rahmen einer Therapie soll der Fokus auf folgende Themengebiete gelegt werden:

- Sammeln von Gründen für das Leben, die in vergangenen suizidalen Krisen wahrnehmbar gewesen sind. Es ist nötig zu überprüfen, ob diese Gründe gegenwärtig auch hilfreich sind.

- In Phasen geringer suizidaler Einengung bzw. bei Wohlergehen sollen Menschen/Interessen/Faktoren gesammelt werden, für die es sich zu leben lohnt bzw. mit denen/durch die Patient_innen schon schöne Erlebnisse oder einen angenehmen Zustand erlebt haben. Hier kann eine Palette von Dingen aufgezählt werden, vom Geruch von frisch gemahlenem Kaffee über (Paten-)Kinder $\mathrm{zu}$ verehrten Künstler_innen, aktivistischen Tätigkeiten, Neugier auf ..., Wärme der ersten Sonnenstrahlen nach bedeckten Tagen, etc.

- Erarbeiten von Situationen, die Patient_innen als gefährdend für den Lebenswillen einstufen. Bewusstheit über Situationen erlangen, in denen sich Patient_innen befinden. Als Basis dafür scheinen folgende Aspekte wichtig:

- Aufbau bzw. Intensivierung der Wahrnehmungsfähigkeit die eigene Befindlichkeit betreffend.

- Üben der Wahrnehmungsfähigkeit in der Therapie und selbstständig in Situationen, in denen sich Patient_innen nicht suizidal erleben (etwa beim Geschirrwaschen, Nägel schneiden, Essen, Kämmen der Haare des Kindes/des Haustiers, etc.)

- Erlernen von Distanzierungstechniken.

- Sammeln von Faktoren (alle Sinne ansprechen), die den Patient_innen in Krisen gut getan/geholfen haben bzw. wie diese überstanden/überlebt wurden.

- Erarbeiten von bereits erreichten Veränderungen in Bezug auf die krisenhafte(n) Situation(en). Etwa neue Gedanken(gänge), Veränderungen der Leistungsanforderung, Veränderung der Bedeutsamkeit der Meinung anderer, selbstfürsorgliche Handlungen, etc.

- Erinnern von Kindheitsträumen.

- Erarbeiten von Zukunftsträumen/-visionen.

- Erarbeiten von Vorstellungen über sich selbst in 1/5/10 Jahren. 
Abschließend kann festgehalten werden, dass bei der Anwendung der Implikationen an erster Stelle die Sinnhaftigkeit für Patient_innen sichtbar werden muss. Die Erarbeitung soll innerhalb einer wohlwollenden Atmosphäre, in intensiver Beziehungsarbeit erfolgen, die getragen ist von Intersubjektivität und Ko-respondenz. Denn Vereinbarungen können nur so wirksam sein, wie die vorhandene Beziehung (Jacobs et al. 2010; Lazic et al. 2015; Page und King 2008).

Ziel der Beschäftigung mit den genannten Schwerpunkten ist die Steigerung des Souveränitätsgefühls sowie ein Bewusstwerdungsprozess über die eigene Handlungsfähigkeit - im Gegensatz zu Gefühlen von Ohnmacht, Einengung und ausgeliefert sein. Die Patient_innen werden als Expert_innen gesehen. Wertschätzung der eigenen Entwicklung gegenüber soll unbedingt Platz finden.

An Klonsky (2019) und Franklin et al. (2017) angelehnt, muss an dieser Stelle wiederholt darauf hingewiesen werden, dass die Voraussage für suizidale Handlungen schwer einschätzbar ist und auch diese genannten Implikationen für die Praxis nicht als Versicherung gesehen werden dürfen. Eher können sie für Zeitgewinn und den Fortgang der Therapie stehen und damit einhergehend den Aufbau bzw. die Intensivierung einer tragfähigen therapeutischen Beziehung fördern.

Merke: Das Thema Suizidgedanken und -impulse muss weiterhin im Rahmen einer Therapie an- und besprechbar bleiben. Die Behandler_innen sind angehalten, eine regelmäßige Befundung der Suizidalität vorzunehmen, denn Suizidalität verändert sich schnell. Die Intervention darf nicht als rechtliche Versicherung missverstanden werden.

Interessenkonflikt L. Gerstl gibt an, dass kein Interessenkonflikt besteht.

Open Access Dieser Artikel wird unter der Creative Commons Namensnennung 4.0 International Lizenz (http:// creativecommons.org/licenses/by/4.0/deed.de) veröffentlicht, welche die Nutzung, Vervielfältigung, Bearbeitung, Verbreitung und Wiedergabe in jeglichem Medium und Format erlaubt, sofern Sie den/die ursprünglichen Autor(en) und die Quelle ordnungsgemäß nennen, einen Link zur Creative Commons Lizenz beifügen und angeben, ob Änderungen vorgenommen wurden.

\section{Literatur}

Aldenhoff, J. (2017). Der Zugang zum Patienten - Anamneseund Befunderhebung. In W. Hewer, W. Rössler \& T. Messer (Hrsg.), Die psychiatrische Notfallmedizin. Management und Therapie (3. Aufl.S. 11-24). München: Elsevier.

Brodsky, B. S., Spruch-Feiner, A., \& Stanley, B. (2018). The Zero Suicide Model: Applying Evidence-Based Suicide Prevention Practices to Clinical Care. Frontiers in Psychiatry, 9(33), 1-7.

Bronisch, T. (2014). Der Suizid. Ursachen, Warnsignale, Prävention (6. Aufl.). München: C.H:Beck.

Bronisch, T. (2017). Suizidalität. In W. Hewer, W. Rössler \& T. Messer (Hrsg.), Die psychiatrische Notfallmedizin. Ma- nagement und Therapie (3. Aufl. S. 165-174). München: Elsevier.

Bryan, J.C., Mintz, J., Clemans, A.T., Leeson, B., Burch, T.S., Williams, R. S., et al. (2017). Effect of crisis response planning vs. contracts for safety on suicide risk in US Army soldiers: a randomized clinical trial. Journal of affective disorders, 212,64-72.

Dorrmann, W. (2009). Suizid. Therapeutische Interventionen bei Selbsttötungssabsichten (6. Aufl.). Stuttgart: Klett-Cotta.

Drew, B. L. (2001). Self-harm behavior and no-suicide contracting in psychiatric inpatient settings. Archives of Psychiatric Nursing, 15(3), 99-106.

Drye, R. C., Goulding, R. L., \&Goulding, M. E. (1973).No-suicide decisions: Patient monitoring of suicidal risk. American Journal of Psychiatry, 130(2), 171-174.

Edwards, S., \& Harries, M. (2007). No-suicide contracts and no-suicide agreements: a controversial life. Australasian Psychiatry, 15(6), 484-489.

Edwards, S. J., \& Sachmann, M.D. (2010). No-suicide contracts, no-suicideagreements, and no-suicideassurances. Astudy of their nature, utilization, perceived effectiveness, and potential to cause Harm. Crisis, 31(6), 290-302.

Franklin, J.C., et al. (2017). Risk factors for suicidal thoughts and behaviors: A meta-analysis of 50 years of research. Psychological Bulletin, 143(2), 187-232.

Jacobs, D. G., et al. (2010). Practice guideline for the assessment and treatment of patients with suicidal behaviors. https://www.psychiatry.org/File\%20Library/ Psychiatrists/Practice/Clinical\%20Practice\%20 Guidelines/suicide.pdf.Zugegriffen:24.Okt. 2018.

Kelly, K.T., \& Knudson, M.P. (2000). Are no-suicide contracts effective in preventing suicide in suicidal patients seen by primary care physicians? Archives of Family Medicine, 9, $1119-1121$.

Kind, J. (2011). Suizidal. Die Psychoökonimie einer Suche (5. Aufl.). Göttingen:Vandenhoeck\& Ruprecht.

Klonsky, E. D. (2019). The role of theory for understanding and preventing suicide (but not predicting it): A commentary on Hjelmeland and Knizek. Death Studies. https://doi. org/10.1080/07481187.2019.1594005.

Lazic, S., Gaudlitz, K., Hättenschwiler, J., \& Modestin, J. (2015). Umgang mit dem suizidalen Patienten in der ambulanten Praxis. Therapeutische Umschau, 72(10), 657-663.

Lee, J.B., \& Bartlett, M.L. (2005). Suicide prevention: critical elements for managing suicidal clients and counselor liability without the use of a no-suicide contract. Death Studies, 29(9), 847-865.

Leitner, A. (2011). Psychotherapie auf dem Weg zur Professionalisierung. Geschichte, Ordnungsmodell, Wirkungen und Neben-Wirkungen. In M. Kierein \& A. Leitner (Hrsg.), Psychotherapie und Recht(S. 85-136). Wien: Facultas.

Page, S.A., \& King, M.C. (2008). No-suicide agreements: Current practices and opinions in a Canadian urban health region. The Canadian Journal of Psychiatry, 53(3), 169-176.

Range, L. M., et al. (2002). No-suicide contracts: an overview and recommendations. Death Studies, 26(1), 51-74.

Reichel, R. (2018). Vom Sinn des Sterbens: Gedanken und Anregungen für den Umgang mit Sterben und mit Sterbenwollen. Wien: Facultas.

Reid, W.H. (1998). Promises, promises: Don't rely on patients' nosuicide/no-violence 'contracts'. Journal of Practice Psychiatry and Behavioral Health, 4,316-318.

Schiller, M. (2011). Die Arzthaftung für Behandlungsfehler im Rahmen psychiatrischer Behandlungen. GesundheitsRecht, 10(1), 8-17. 
Sonneck, G. (2001).Zur Alltagspraxis im Umgang mit Suizidgefährdeten. In R. Hutterer-Krisch (Hrsg.), Fragen der Ethik in der Psychotherapie. Konfliktfelder, Machtmißbrauch, Berufspflichten (2.Aufl.S.313-327). Wien: Springer.

Sonneck, G., Kapusta, N., Tomandl, G., \& Voracek, M. (Hrsg.). (2016). Krisenintervention und Suizidverhütung(3.Aufl.). Wien: Facultas.

Stanford, E. J., Goetz, R. R., \& Bloom, J. D. (1994). The no-harm contract in the emergency assessment of suicidal risk. The Journal of Clinical Psychiatry, 55, 344-348.

Stanley, B., \& Brown, G. K. (2012). Safety planning intervention: a brief intervention to mitigate suicide risk. Cognitive and Behavioral Practice, 19, 256-264.
Stanley, B., Green, K. L., Ghahramanlou-Holloway, M., Brenner, L.A., \& Brown, G. K. (2017). The construct and measurement of suicide-related coping. Psychiatry Research, 258, 189-193.

Wolfersdorf, M. \&Etzersdorfer, E. (2011). Suizid und Suizidprävention. Stuttgart: Kohlhammer.

Hinweis des Verlags Der Verlag bleibt in Hinblick auf geografische Zuordnungen und Gebietsbezeichnungen in veröffentlichten Karten und Institutsadressen neutral. 